\title{
DCE-MRI biomarkers of tumour heterogeneity predict CRC liver metastasis shrinkage following bevacizumab and FOLFOX-6
}

\section{JPB O'Connor, 1,2,5, CJ Rose', ${ }^{1,5}$ A Jackson', Y Watson', S Cheung', F Maders', BJ Whitcher ${ }^{3}$, C Roberts', GA Buonaccorsi', G Thompson', AR Clamp ${ }^{4}$, GC Jayson ${ }^{4}$ and GJM Parker'}

'Imaging Science, Proteomics and Genomics Research Group, School of Cancer and Enabling Sciences, University of Manchester, Manchester Academic Health Sciences Centre, Oxford Road, Manchester MI 3 9PT, UK; ${ }^{2}$ Department of Radiology Christie Hospital, Wilmslow Road, Manchester M20 4BX, UK; ${ }^{3}$ Clinical Imaging Centre, GlaxoSmithKline, Hammersmith Hospital, Imperial College London, Du Cane Road, London WI 2 OHS, UK; ${ }^{4}$ Cancer Research UK Department of Medical Oncology, Christie Hospital, Wilmslow Road, Manchester M20 4BX, UK

BACKGROUND: There is limited evidence that imaging biomarkers can predict subsequent response to therapy. Such prognostic and/or predictive biomarkers would facilitate development of personalised medicine. We hypothesised that pre-treatment measurement of the heterogeneity of tumour vascular enhancement could predict clinical outcome following combination anti-angiogenic and cytotoxic chemotherapy in colorectal cancer (CRC) liver metastases.

METHODS: Ten patients with 26 CRC liver metastases had two dynamic contrast-enhanced MRI (DCE-MRI) examinations before starting first-line bevacizumab and FOLFOX-6. Pre-treatment biomarkers of tumour microvasculature were computed and a regression analysis was performed against the post-treatment change in tumour volume after five cycles of therapy. The ability of the resulting linear model to predict tumour shrinkage was evaluated using leave-one-out validation. Robustness to inter-visit variation was investigated using data from a second baseline scan.

RESULTS: In all, $86 \%$ of the variance in post-treatment tumour shrinkage was explained by the median extravascular extracellular volume $\left(v_{e}\right)$, tumour enhancing fraction $\left(E_{F}\right)$, and microvascular uniformity (assessed with the fractal measure box dimension, $d_{0}$ ) $\left(R^{2}=0.86, P<0.00005\right)$. Other variables, including baseline volume were not statistically significant. Median prediction error was $12 \%$. Equivalent results were obtained from the second scan.

CONCLUSION: Traditional image analyses may over-simplify tumour biology. Measuring microvascular heterogeneity may yield important prognostic and/or predictive biomarkers.

British Journal of Cancer (201 I) 105, 139- 145. doi: I0.1038/bjc.20 I I.191 www.bjcancer.com

Published online 14 June 2011

(c) 20I I Cancer Research UK

Keywords: angiogenesis; biomarker; heterogeneity; MRI; outcome; personalised medicine

There is considerable interest in developing pre-treatment biomarkers of microvascular structure and function that predict subsequent therapeutic response (O'Connor et al, 2008). Development and validation of such biomarkers will be essential if personalised therapy is to become a reality (Meyer et al, 2009).

Tumour size is an important factor in staging some solid tumours, selecting treatment options, and in predicting clinical outcome (Edge et al, 2010). However, for some solid tumours, size has little relevance to tumour stage and the link between pretreatment tumour size and outcome is complex, with no clear relationship between the two (Grigsby et al, 1999; Foulkes et al, 2008; Klatte et al, 2008).

Tumour function may also predict outcome, particularly in the setting of novel adjuvant therapies. Techniques such as ${ }^{18} \mathrm{~F}$-fluorodeoxyglucose positron emission tomography $\left({ }^{18} \mathrm{~F}-\mathrm{FDG}-\right.$ PET) and dynamic contrast-enhanced MRI (DCE-MRI) offer the

\footnotetext{
*Correspondence: Dr JPB O'Connor;

E-mail: james.o'connor@manchester.ac.uk

${ }^{5}$ These authors contributed equally to this work.

Received I8 October 20 I0; revised 20 April 20 I ; accepted 5 May 20 II; published online I4 June 201 |
}

opportunity to study tumour pathophysiology (O'Connor et al, 2008). For example, simple summary values such as high baseline ${ }^{18}$ FDG-PET standardised uptake value (Dimitrakopoulou-Strauss et al, 2004; de Geus-Oei et al, 2006) and high baseline DCE-MRI volume transfer constant $\left(K^{\text {trans }}\right)$ (George et al, 2001) before therapy have shown statistically significant relationships with beneficial clinical outcome following various cytotoxic treatments in patients with colorectal cancer (CRC). However, current evidence that these biomarkers accurately predict clinical outcome is limited (O'Connor et al, 2007a; Kinahan et al, 2009).

This relative lack of success has fuelled interest in alternative biomarkers of microvascular structure and function that may better serve as predictive and/or prognostic biomarkers. There is emerging evidence that tumours are biologically complex structures exhibiting marked spatial variation in angiogenesis (Kumar et al, 1998; Eberhard et al, 2000), hypoxia (Picchio et al, 2008), cell death (Gonzalez-Garcia et al, 2002), and glucose metabolism (Schroeder et al, 2005). The presence and degree of heterogeneity may be an important determinant of cancer metastatic potential (Fidler, 1987) and response to therapy (Casanovas et al, 2005). Despite this, measurement of tumour heterogeneity is largely ignored in radiological practice. 
Previous imaging studies have provided evidence of a relationship between the spatial heterogeneity of image biomarkers of the tumour vasculature (such as $T_{1}$ signal change, $K^{\text {trans }}$, blood volume, and Hounsfield units) and therapeutic response (Jackson et al, 2007). Several differing approaches have been studied, including histogram analysis (Chang et al, 2004) and measuring the proportion of the tumour that enhances (O'Connor et al, $2007 \mathrm{~b})$. Both of these approaches summarise the distribution of tumour functional properties but ignore the spatial location of individual tumour voxels (and their vascular, metabolic, or other features) and the relationship of one voxel to another within a lesion. Alternative approaches such as texture (El Naqa et al, 2009) or fractal analysis (Dzik-Jurasz et al, 2004; Rose et al, 2007; Goh et al, 2009; Alic et al, 2011) quantify the overall spatial complexity of a tissue and retain information regarding the spatial arrangement of voxels within a tumour. In this study, we tested the hypothesis that tumour measurements of microvascular function and heterogeneity computed from pre-treatment DCE-MRI data would predict tumour shrinkage following combination therapy with anti-angiogenic and cytotoxic chemotherapy.

\section{MATERIALS AND METHODS}

\section{Study design}

Retrospective analysis was performed on DCE-MRI data collected between July 2006 and October 2007 in 10 patients with CRC liver metastases. Patients were originally recruited for an investigator led study that examined the temporal action of bevacizumab during a single cycle of therapy (O'Connor et al, 2009). Ethical approval was granted by the local Research Ethics Committee and informed consent was obtained. All patients received single agent $10 \mathrm{mg} \mathrm{kg}^{-1}$ bevacizumab (cycle 1) followed every 2 weeks by $10 \mathrm{mg} \mathrm{kg}^{-1}$ bevacizumab plus FOLFOX-6 (oxaliplatin/5FU/leucovorin) for 2 months (cycles $2-5$ ) as first-line treatment.

\section{Patient recruitment}

Patients with histologically proven primary epithelial CRC, aged $\geqslant 18$ years, with an Eastern Cooperative Oncology Group score between 0 and 2, and life expectancy of at least 3 months were eligible. Enrolment of patients who required first-line treatment for metastatic disease was consecutive. Inclusion criteria were presence of a measurable lesion $\geqslant 2 \mathrm{~cm}$ on previous imaging; adequate liver, renal, and haematologic function; normal coagulation (prothrombin time and activated partial thromboplastin time); normal ECG.

Exclusion criteria were previous treatment with vascular endothelial growth factor (VEGF) inhibitors or cytotoxic chemotherapy; contraindications to VEGF inhibitors; exposure to any other investigational drug (within the last 4 weeks) or concurrent therapy likely to influence the vasculature on imaging; pregnant or breast-feeding women; previous clinically significant haemorrhage, thrombosis, or cardiovascular disease within the last 6 months; proteinuria; contraindication to MRI.

\section{MRI data acquisition}

Patients were examined on a 1.5 -T Philips Intera system (Philips Medical Systems, Best, The Netherlands). Each patient was scanned twice before treatment, to allow measurement reliability to be assessed. In one patient (with three tumours), data were only available from one pre-treatment scan. The field of view (FOV) was centred on the liver. In each examination, $T_{1}$-weighted fast field echo images ( $\mathrm{TR}=10 \mathrm{~ms}, \mathrm{TE}=4.6 \mathrm{~ms}, \alpha=15^{\circ}$ ) and $T_{2}$-weighted single shot turbo spin echo images $(\mathrm{TR}=606.5 \mathrm{~ms}, \mathrm{TE}=80 \mathrm{~ms}$, $\alpha=90^{\circ}$ ) were acquired. Both sequences employed FOV $375 \times 375 \mathrm{~mm}^{2}$, matrix $256 \times 256$ with a 4 -mm slice thickness.
For the DCE-MRI series, $753 \mathrm{D}$ axial volumes were acquired consecutively $\left(\mathrm{TR}=4.0 \mathrm{~ms}, \mathrm{TE}=0.82 \mathrm{~ms}, \alpha=20^{\circ}\right.$, one signal average, FOV of $375 \times 375 \mathrm{~mm}^{2}$, matrix $128 \times 128$; in-plane voxel size $2.93 \times 2.93 \mathrm{~mm}^{2}$ ) following calculation of baseline $T_{1}$ using the variable flip angle method (Fram et al, 1987) $\left(\alpha=2^{\circ} / 10^{\circ} / 20^{\circ}\right.$; four signal averages; identical TR, TE, imaging matrix, and slice thickness). Temporal resolution was $4.97 \mathrm{~s}$. On the sixth dynamic time point, $0.1 \mathrm{mmol} \mathrm{kg}^{-1}$ of Gadodiamide (Omniscan GE Medical Systems, Amersham, UK) was administered intravenously through a Spectris MR power injector (Medrad Inc., Indianola, PA, USA) at $3 \mathrm{ml} \mathrm{s}^{-1}$, followed by a saline flush. Slice thickness was $4 \mathrm{~mm}$ for small target lesions or $8 \mathrm{~mm}$ for larger lesions, giving superiorinferior coverage of either 100 or $200 \mathrm{~mm}$. Images were acquired during gentle free breathing.

\section{Calculation of tumour volume and summary DCE-MRI statistics}

Quality control was applied to reduce error in all image parameters. The impact of motion was assessed and tumours for which parameter estimates would be unreliable were rejected. The level of bulk motion was assessed for each tumour by first extracting an averaged time series plot for each tumour region of interest (ROI) on each slice in the imaging volume and then by visual assessment of the dynamic time series images. In- and through-plane motion was investigated and a categorical score was assigned for each tumour based on the evaluations of bulk motion (slight motion $=1$, moderate motion $=2$, significant motion $=3$, and severe motion $=4$ ). Tumours with a motion assessment score of 3 or 4 were excluded.

Three-dimensional ROIs were defined on coregistered highresolution $T_{1^{-}}$and $T_{2}$-weighted sequences. In some patients, multiple lesions were defined. To quantify microvascular characteristics, the extended Tofts version of the Kety model (Tofts, 1997) was fitted to the DCE-MRI time series at each enhancing tumour voxel (see below) using in-house software, as given by

$$
C_{t}(t)=v_{\mathrm{p}} C_{\mathrm{p}}(t)+K^{\mathrm{trans}} \int_{0}^{t} C_{\mathrm{p}}\left(t^{\prime}\right) \exp \left(\frac{-K^{\operatorname{trans}}\left(t-t^{\prime}\right)}{v_{\mathrm{e}}}\right) \mathrm{d} t^{\prime}
$$

where $C_{t}(t)$ is the concentration of contrast agent at time $t$ in each voxel and $C_{\mathrm{p}}(t)$ is the concentration of contrast agent in the arterial blood plasma (i.e., the arterial input function, which was determined using a previously published population AIF technique (Parker et al, 2006)). The extended Tofts model is only valid in tumour regions in which there is a measurable signal change due to the presence of contrast agent. To identify those voxels, a previously published method was used in which pre- and post-injection concentration values are statistically compared (O’Connor et al, 2009).

Voxel-wise analysis was performed allowing estimates of the median bulk transfer coefficient $\left(K^{\text {trans }}\right.$; units $\min ^{-1}$; Figure $\left.1 \mathrm{~A}\right)$, mean fractional plasma volume $\left(v_{\mathrm{p}}\right.$; unitless) and median fractional volume of the extravascular extracellular space $\left(v_{\mathrm{e}}\right.$; unitless).

\section{Calculation of heterogeneity statistics}

The following three types of heterogeneity statistic were derived:

1. Enhancing fraction $\left(E_{\mathrm{F}}\right.$, defined as the ratio of the number of enhancing voxels to total tumour voxels; $E_{\mathrm{F}}=N_{\mathrm{E}} / N_{\mathrm{T}}$ ) was calculated to characterise the overall level of tumour perfusion (Figure 1B). Voxels with pre-contrast and post-contrast agent time series that had significantly different distributions (where $P<0.05$ on Mann-Whitney Wilcoxon rank-sum test) were classified as enhancing (O'Connor et al, 2007b). 

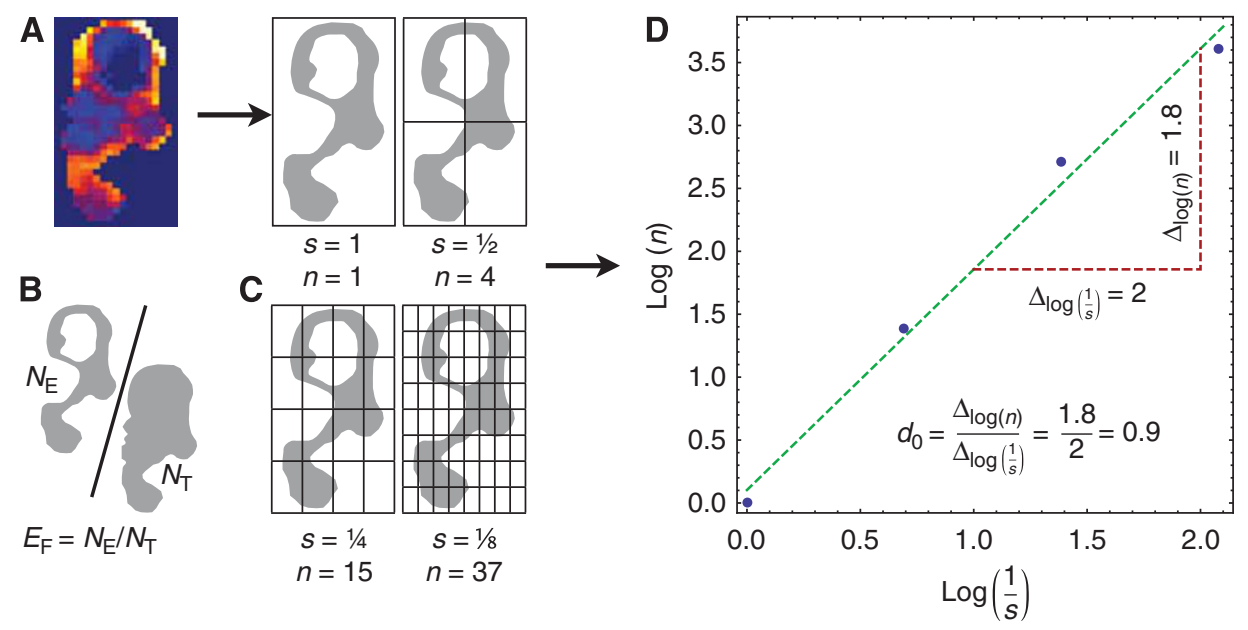

Figure I Derivation of a thresholded parameter map to enable calculation of enhancing fraction $\left(E_{\mathrm{F}}\right)$ and box dimension $\left(d_{0}\right)$. (A) $K^{\text {trans }}$ map across a single slice within a CRC liver metastasis shows marked spatial heterogeneity. (B) A criterion is applied to the contrast agent concentration time series to identify enhancing voxels $\left(N_{E}\right)$ and the resultant map is shown. $E_{F}$ is calculated as the ratio of $N_{E}$ to the number of tumour voxels ( $\left.N_{T}\right)$. (C) A box surrounding the object defined by the enhancing voxels is successively divided, defining a range of scales (s) at which the number of boxes containing a part of the object is counted $(n)$. (D) $d_{0}$ is the slope of the line of best fit through the points $(\log n, \log \mid / s)$ and quantifies the space filling properties of the parameter map.

2. Standard deviations of the voxel-wise $K^{\text {trans }}, v_{\mathrm{e}}$, and $v_{\mathrm{p}}$ measurements were calculated for the enhancing portion of each tumour.

3. Fractal summaries (measures of microvascular structure and function that consider spatial information) were derived from the DCE-MRI data. The calculation of one of these fractal measures, box dimension $\left(d_{0}\right)$, is illustrated (Figures $1 \mathrm{C}$ and $\mathrm{D}$ ), where a binary image corresponding to enhancing tumour voxels is iteratively subsampled by a factor of two to define binary images at a range of scales; at each scale, the enhancing voxels are counted and $d_{0}$ is calculated as the rate of change in the number of enhancing voxels with respect to scale (with both quantities considered on logarithmic scales). In addition, fractal measures based on the information dimension $\left(d_{1}\right)$ and correlation dimension $\left(d_{2}\right)$ were calculated. These latter metrics retain magnitude values of $K^{\text {trans }}, v_{\mathrm{e}}$, and $v_{\mathrm{p}}$ and are described in detail elsewhere (Rose et al, 2009).

\section{Evaluation of tumour shrinkage}

$\mathrm{X}$-ray computed tomography (CT) examination of the abdomen and pelvis was performed at baseline within $72 \mathrm{~h}$ of the baseline MRI and at the end of cycle 5 (EC5) to evaluate clinical response. Patients were imaged on a LightSpeed Plus CT scanner (GE Medical Systems), with typical clinical helical acquisition variables (tube voltage $120 \mathrm{kV}$, tube current $40 \mathrm{~mA}$ ). Images were acquired following intravenous injection of $200 \mathrm{ml}$ Omnipaque-140 (GE Medical Systems) and reformatted to produce contiguous $5 \mathrm{~mm}$ slices with no overlap. Tumour volumes were measured (in $\mathrm{mm}^{3}$ ) and the remaining tumour volume (\%) from baseline to EC5 was calculated by comparison with the pre-treatment tumour volume.

\section{Statistical analysis}

Percentage of remaining tumour volume at EC5 was modelled as a linear function of the pre-treatment summary statistics described above. Before modelling, variables were transformed as appropriate to improve linearity (e.g., by taking logarithms). In a preliminary analysis, a mixed-effects model was used to explore potential within-patient clustering since some patients had more than one tumour. However, no statistically significant evidence for such effects was found. Subsequently, tumours were treated independently.

Stepwise errors-in-variables regression was used to model the percentage of remaining tumour volume based on the pretreatment volume, summary DCE-MRI statistics, and heterogeneity statistics using data from the first pre-treatment scan. The regression was repeated using the data from the second scan to investigate robustness to inter-visit variation. In the above analyses, the missing data for one patient's first pre-treatment scan (three tumours) were dealt with using list-wise deletion or imputation (from the second scan) as appropriate, with the aim of maximising the amount of data available, while minimising bias.

The ability of the linear model to predict tumour response was evaluated using two leave-one-out analyses. In the first analysis, each tumour was left out in turn; the coefficients on each variable were computed-by applying errors-in-variables regression to the left-in tumours' data-and used to predict the response of the leftout tumour. In the second analysis, the data for each patient was left out in turn (allowing us to further investigate potential intrapatient clustering effects); the coefficients on each variable were computed-by applying errors-in-variables regression to the leftin patients' data-and used to predict the responses for the tumours in the left-out patients.

Prediction error was quantified using the absolute difference between the actual and predicted percentage of remaining tumour volume. A cumulative distribution function (CDF) of prediction error was plotted for each leave-one-out analysis; a CDF permits estimation of the proportion of predictions that would be expected to be less than or equal to a given prediction error. Bland-Altman plots were formed to assess the agreement between actual and predicted percentage of the remaining tumour volume.

Statistical modelling was performed using Stata/IC version 10.1 (Stata Corporation, College Station, TX, USA) and leave-one-out analysis was performed using Mathematica version 7.0.1 (Wolfram Research, Champaign, IL, USA).

\section{RESULTS}

The mean patient age was 68.3 years (range 61-78 years; eight males; two females). All patients completed therapy to EC5. Two patients achieved partial responses; seven had stable disease; one 
Table I Result of the errors-in-variables regression shows: the model's $F$ statistic, $P$, and $R^{2}$ values

$\begin{array}{ll}F_{3,22} & 25.86 \\ P & \leqslant 0.00005 \\ R^{2} & 0.86\end{array}$

\begin{tabular}{lcrccc} 
Variable & Coefficient & $\boldsymbol{T}$ & $\boldsymbol{P}>|\mathbf{t}|$ & \multicolumn{2}{c}{$\mathbf{9 5 \%} \mathbf{C l}$ on coefficient } \\
\hline$v_{\mathrm{e}}$ & -147.08 & -3.37 & 0.003 & -237.49 & -56.67 \\
$E_{\mathrm{F}}$ & -2.35 & -8.46 & $\leqslant 0.0005$ & -2.93 & -1.78 \\
$d_{0}$ & $\mid 56.10$ & 4.04 & 0.001 & 75.91 & 236.30 \\
Constant & -47.19 & -0.68 & 0.506 & -191.83 & 97.45 \\
\hline
\end{tabular}

Abbreviations: $d_{0}=$ fractal measure box dimension; $E_{\mathrm{F}}=$ enhancing fraction; $v_{\mathrm{e}}=$ median extravascular extracellular space volume. The variables listed were significant in the final model (corresponding coefficients, $t$ statistics, $P$ values, and $95 \%$ confidence intervals (Cls) are provided). The constant term in the linear model is also included.

had disease progression by RECIST 1.0 criteria. In all, 26 tumours were identified in the 10 patients (mean 2.6, median 2.5).

The final errors-in-variables regression analysis modelled tumour response in terms of the following pre-treatment biomarkers: median $v_{\mathrm{e}}, E_{\mathrm{F}}$, and $d_{0}$ (details in Table 1). This model explained $86 \%$ of the variance in tumour response $(95 \%$ confidence interval 77-94\%). When the regression was repeated for the second pre-treatment scan data, no significant differences between the coefficients estimated for each variable, or between the $R^{2}$ values, were found; however, median $v_{\mathrm{e}}$ was not quite significant within this second model $(P=0.07)$. This suggests that the identified variables and the underlying model are robust to typical inter-visit variation. Scatter plots showing the relationships between the retained variables and percentage remaining tumour volume are provided in Figure 2.

The results of the two leave-one-out analyses suggest that tumour response can be predicted with an error of no more than $12 \%$ in $50 \%$ of cases, and with an error of no more than $31 \%$ in $80 \%$ of cases (Figure 3 ). No difference was observed between the leave-one-out analysis that treated tumours independently and that grouped tumours at the patient level, indicating no evidence for intra-patient clustering.

The Bland-Altman plots for the leave-one-tumour- and leaveone-patient-out predictions were very similar, with the differences (vertical axes) having almost identical mean $(-0.04 \%)$ and standard deviation $(30 \%)$ (Figure 4$)$. In one tumour, the remaining tumour volume was predicted particularly poorly (the model dramatically underestimates the actual change). There was no statistically significant relationship between the means and differences.

\section{DISCUSSION}

In this study, we have investigated the relative value of pretreatment biomarkers of the microvasculature in explaining the percentage of remaining tumour volume resulting from combined bevacizumab and FOLFOX-6 therapy. We hypothesised that tumour size change could be explained by baseline image data, but that simple measures of size or function (used individually) may lack predictive power.

In this data set, $86 \%$ of the variance in the outcome measure (percentage remaining tumour volume EC5) was explained by combining various pre-treatment imaging biomarkers. Importantly, robustness to inter-visit variation was validated by a second data set derived from the same tumours. Of note, pre-treatment tumour volume was not found to be a statistically significant determinant of subsequent change in tumour volume following treatment. However, three variables $\left(v_{\mathrm{e}}, E_{\mathrm{F}}\right.$, and $\left.d_{0}\right)$ were
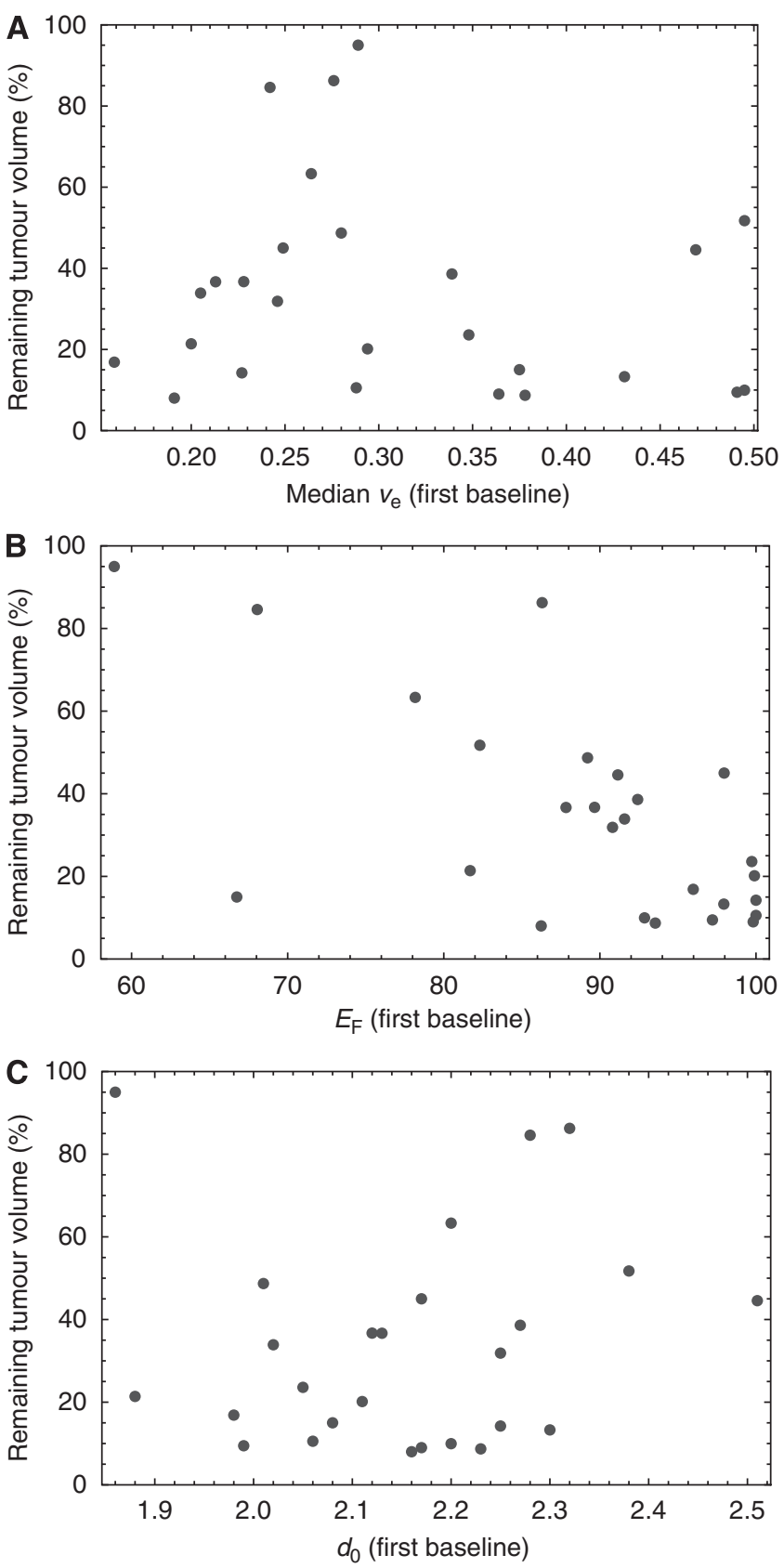

Figure 2 Scatter plots showing the relationship between $(\mathbf{A})$ median $v_{\mathrm{e}}$ (B) $E_{F}$, and $(\mathbf{C}) d_{0}$ and remaining tumour volume (\%).

statistically significant within the model and provide complementary types of information about the tumour environment. These results are congruent with other studies, which report that multiparametric image analyses may be better at predicting clinical outcome (Dimitrakopoulou-Strauss et al, 2004, 2010), compared with more traditional analyses based around a single parameter.

Median $v_{\mathrm{e}}$ is an estimate of the extracellular extravascular space affected by factors including cell size, number, and packing density. It also represents a direct estimate of the distribution space to which a contrast agent or drug can be delivered. Different studies have reported variably either decrease or increase in median $v_{\mathrm{e}}$ in small numbers of patients following anti-vascular therapy. No study has reported how this parameter may relate to a measure of clinical outcome. In this study, high median $v_{\mathrm{e}}$ was 


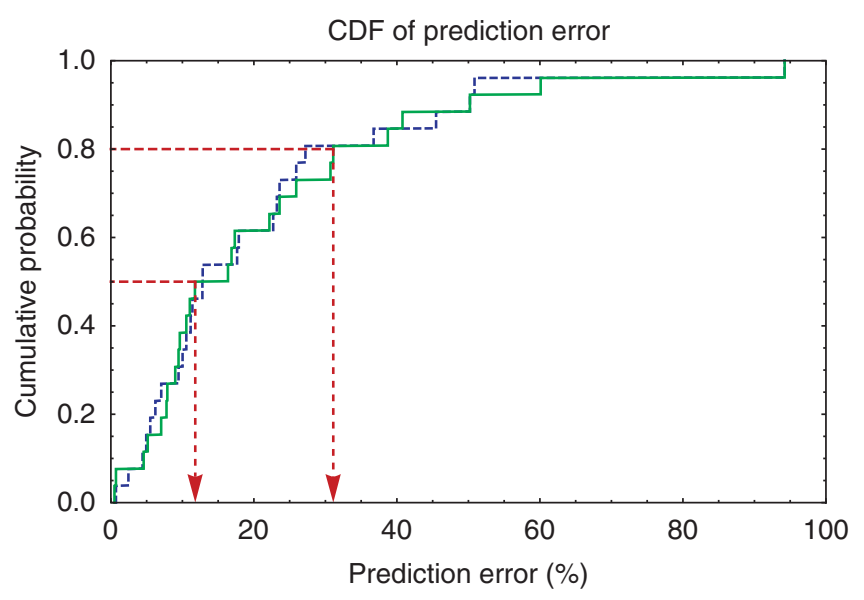

Figure 3 Cumulative distribution functions of prediction error for the leave-one-tumour-out (dashed blue) and leave-one-patient-out (solid green) analyses. Red lines show prediction error for $50 \%$ and $80 \%$ of all cases.
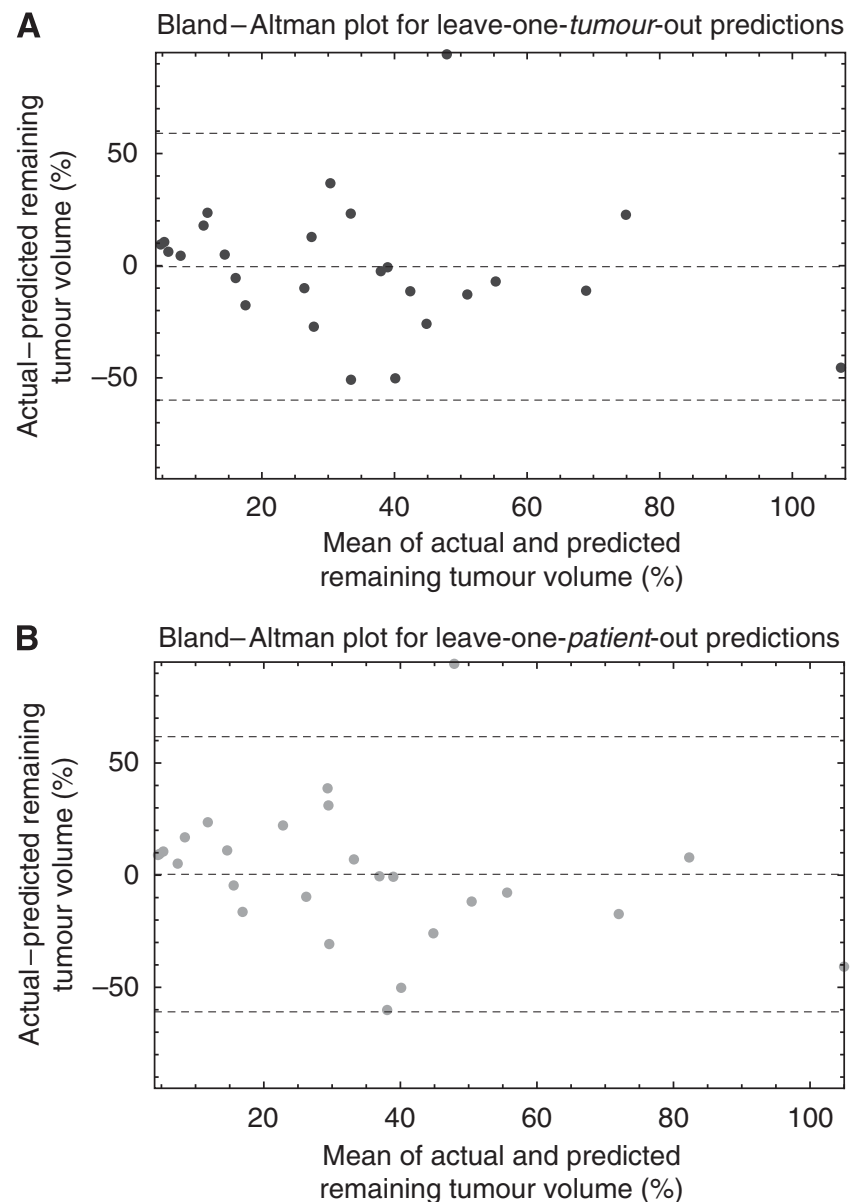

Figure 4 Bland-Altman plots for (A) leave-one-tumour-out and (B) leave-one-patient-out predictions, showing the mean difference between the actual and predicted changes in volume, and that mean \pm 2 s.d. of the differences.

associated with greater tumour shrinkage, which may indicate the potential for greater extravasations of chemotherapy and bevacizumab into the extracellular extravascular space.
Two measures of tumour vascular heterogeneity $-E_{\mathrm{F}}$ and $d_{0}-$ were also statistically significant in this study. $E_{\mathrm{F}}$ is the ratio of enhancing tumour voxels to overall tumour voxels. As such, it is a crude indicator of heterogeneity by quantifying the proportion of a tumour that has demonstrable delivery and retention of contrast agent. For example, a tumour with cystic or necrotic components has a lower $E_{\mathrm{F}}$ than a predominantly solid lesion. The parameter is repeatable with a low coefficient of variation (O'Connor et al, 2009) and is sensitive to the therapeutic effect of anti-angiogenic and antivascular compounds (Galbraith et al, 2003; Robinson et al, 2003; O'Connor et al, 2009). In this study, high $E_{\mathrm{F}}$ was associated with better tumour response. This is consistent with identifying tumours with a high proportion of well-perfused tissue that receive substantial penetration of systemically administered agents.

Previous studies have reported that high baseline $E_{\mathrm{F}}$ predicted poor response to cytotoxic chemotherapy in epithelial ovarian cancer (O'Connor et al, 2007b) and radiotherapy in cervical cancer (Donaldson et al, 2010), attributed to a high $E_{\mathrm{F}}$ representing a greater amount of neo-angiogenic tumour tissue. This apparent discrepancy may reflect the fact that $E_{\mathrm{F}}$ is a non-specific marker that is influenced by several physiological correlates including flow, permeability, vascular volume, extravascular leakage space, and interstitial pressure. $E_{\mathrm{F}}$ may therefore, be best interpreted relative to normal tissue values rather than absolute values that may indicate prognosis. The type of treatment employed and method of calculating enhancement also vary between studies and alter the relative meaning of a high or low $E_{\mathrm{F}}$.

Enhancing fraction and $v_{\mathrm{e}}$ disregard spatial information; two parameter maps with identical $E_{\mathrm{F}}$ or $v_{\mathrm{e}}$ values can have completely different spatial distributions of either parameter (Rose et al, 2009). For this reason, there has been interest in developing metrics such as box dimension that quantify the spatial heterogeneity present within parametric imaging maps of tumours (Goh et al, 2009). In this study, low box dimension $\left(d_{0}\right)$ was associated with better tumour response. This parameter can be low if there are few enhancing voxels or if enhancing voxels are non-uniformly distributed. The proportion of enhancing voxels is already captured in $E_{\mathrm{F}}$ but $d_{0}$ is also significant, implying that the spatial arrangement of enhancing tissue within a tumour (and therefore the uniformity of drug delivery) is important. As used here, $d_{0}$ reflects not only microvascular uniformity but also depends on tumour shape. Our data are comparable to a study of rectal carcinoma treated by cytotoxic chemotherapy, where a similar parameter calculated from pre-treatment thresholded single slice parameter maps for area under the $\Delta R_{2}{ }^{*}$ curve (where $R_{2}{ }^{*}=1 / T_{2}{ }^{*}$ ) predicted tumour regression after 8 weeks of therapy (Dzik-Jurasz et $a l, 2004)$ and a study of limb sarcomas where fractal dimension distinguished responders from non-responders (Alic et al, 2011).

The CDFs presented in Figure 3 show that in general, percentage remaining tumour volume can be predicted with relatively little error in this particular clinical scenario. This application may be extremely useful for the selection of patients likely to benefit from expensive novel therapies, as it may be possible to identify patients whose tumours that are more or less likely to respond to therapy. The little difference between the CDFs for the leave-one-tumourand leave-one-patient-out analyses, suggest that the ability of the variables identified to predict tumour response cannot easily be explained by the influence of patients with multiple tumours. The resulting model explains a large proportion $(86 \%)$ of the total variance in tumour response and allows tumour response to be predicted with excellent accuracy in the majority of cases. However, while the variables were highly significant within the model, the confidence intervals on their coefficients are wide; a larger sample would be required to estimate these coefficients with more confidence. The model was also robust to inter-visit variation, since the same variables were significant (with the exception of median $v_{\mathrm{e}}$ ), and there were no significant differences between the coefficient or $R^{2}$ values - providing internal validation. 
Our study has four main limitations. First, it was retrospective. Second, the imaging parameters used require significant postprocessing effort to obtain and do not have accepted standardisation. Third, while the biomarkers identified appear to predict shrinkage, they require testing against survival in a larger study. Fourth, respiratory or other patient motion can complicate any image analysis, particularly those performed on a per voxel basis (Orton et al, 2009). Image registration can be used to salvage motion-corrupted data, but requires additional post-processing work, limiting its applicability in clinical settings (Buonaccorsi et al, 2006, 2007). Our results demonstrate that tumour shrinkage can be predicted even in the presence of typical patient motion (however, note that we did reject data that were corrupted by very significant motion). Future work should determine if image registration offers any advantage in the context of predicting tumour shrinkage or survival. Finally, in addition to seeking to improve prediction accuracy, future work should also investigate the use of imaging (or other) information to identify tumours for which the kind of model proposed here would perform poorly.

\section{REFERENCES}

Alic L, van Vliet M, van Dijke CF, Eggermont AM, Veenland JF, Niessen WJ (2011) Heterogeneity in DCE-MRI parametric maps: a biomarker for treatment response? Phys Med Biol 56: 1601-1616

Buonaccorsi GA, O'Connor JP, Caunce A, Roberts C, Cheung S, Watson Y, Davies K, Hope L, Jackson A, Jayson GC, Parker GJ (2007) Tracer kinetic model-driven registration for dynamic contrast-enhanced MRI time-series data. Magn Reson Med 58: 1010 - 1019

Buonaccorsi GA, Roberts C, Cheung S, Watson Y, O'Connor JP, Davies K, Jackson A, Jayson GC, Parker GJ (2006) Comparison of the performance of tracer kinetic model-driven registration for dynamic contrast enhanced MRI using different models of contrast enhancement. Acad Radiol 13: $1112-1123$

Casanovas O, Hicklin DJ, Bergers G, Hanahan D (2005) Drug resistance by evasion of antiangiogenic targeting of VEGF signaling in late-stage pancreatic islet tumors. Cancer Cell 8: 299-309

Chang YC, Huang CS, Liu YJ, Chen JH, Lu YS, Tseng WY (2004) Angiogenic response of locally advanced breast cancer to neoadjuvant chemotherapy evaluated with parametric histogram from dynamic contrast-enhanced MRI. Phys Med Biol 49: 3593-3602

de Geus-Oei LF, Wiering B, Krabbe PF, Ruers TJ, Punt CJ, Oyen WJ (2006) FDG-PET for prediction of survival of patients with metastatic colorectal carcinoma. Ann Oncol 17: 1650-1655

Dimitrakopoulou-Strauss A, Strauss LG, Burger C, Ruhl A, Irngartinger G, Stremmel W, Rudi J (2004) Prognostic aspects of 18F-FDG PET kinetics in patients with metastatic colorectal carcinoma receiving FOLFOX chemotherapy. J Nucl Med 45: $1480-1487$

Dimitrakopoulou-Strauss A, Strauss LG, Egerer G, Vasamiliette J, Mechtersheimer G, Schmitt T, Lehner B, Haberkorn U, Stroebel P, Kasper B (2010) Impact of dynamic 18F-FDG PET on the early prediction of therapy outcome in patients with high-risk soft-tissue sarcomas after neoadjuvant chemotherapy: a feasibility study. J Nucl Med 51: $551-558$

Donaldson SB, Buckley DL, O'Connor JP, Davidson SE, Carrington BM, Jones AP, West CM (2010) Enhancing fraction measured using dynamic contrast-enhanced MRI predicts disease-free survival in patients with carcinoma of the cervix. Br J Cancer 102: 23-26

Dzik-Jurasz A, Walker-Samuel S, Leach MO, Brown G, Padhani AR, George M, Collins DJ (2004) Fractal parameters derived from analysis of DCE-MRI data correlates with response to therapy in rectal carcinoma. Proc Int Soc Magn Reson Med 12: 2503

Eberhard A, Kahlert S, Goede V, Hemmerlein B, Plate KH, Augustin HG (2000) Heterogeneity of angiogenesis and blood vessel maturation in human tumors: implications for antiangiogenic tumor therapies. Cancer Res 60: $1388-1393$

Edge SB, Byrd DR, Compton CC, Fritz AG, Greene FL, Trotti A (2010) AJCC Cancer Staging Handbook, 5th edn, Springer: New York

El Naqa I, Grigsby P, Apte A, Kidd E, Donnelly E, Khullar D, Chaudhari S, Yang D, Schmitt M, Laforest R, Thorstad W, Deasy JO (2009) Exploring
In conclusion, these data provide preliminary evidence that a combination of pre-treatment measures derived from DCE-MRI parameter maps may predict tumour shrinkage in response to combined bevacizumab and cytotoxic chemotherapy in CRC liver metastases, with relatively low error. Although the applicability of these results to other tumours types and metastatic disease in other organs cannot be inferred from the data presented, this type of approach may have value in determining personalised tumour therapy regimes and patient selection. These results encourage the further evaluation of image heterogeneity in cancer studies using MRI, PET, and other techniques, to explore whether similar findings are seen in alternative combinations of patient group, therapy, and imaging technique.

\section{ACKNOWLEDGEMENTS}

JOC was funded by a Cancer Research UK Clinical Research Training Fellowship Grant, ref C19221/A6086. CJR was funded by GlaxoSmithKline to develop the methods used described in this study.

feature-based approaches in PET images for predicting cancer treatment outcomes. Pattern Recognit 42: $1162-1171$

Fidler IJ (1987) Review: biologic heterogeneity of cancer metastases. Breast Cancer Res Treat 9: 17-26

Foulkes WD, Grainge MJ, Rakha EA, Green AR, Ellis IO (2008) Tumor size is an unreliable predictor of prognosis in basal-like breast cancers and does not correlate closely with lymph node status. Breast Cancer Res Treat 117(1): 199-204

Fram EK, Herfkens RJ, Johnson GA, Glover GH, Karis JP, Shimakawa A, Perkins TG, Pelc NJ (1987) Rapid calculation of T1 using variable flip angle gradient refocused imaging. Magn Reson Imaging 5: 201-208

Galbraith SM, Maxwell RJ, Lodge MA, Tozer GM, Wilson J, Taylor NJ, Stirling JJ, Sena L, Padhani AR, Rustin GJ (2003) Combretastatin A4 phosphate has tumor antivascular activity in rat and man as demonstrated by dynamic magnetic resonance imaging. J Clin Oncol 21: $2831-2842$

George ML, Dzik-Jurasz AS, Padhani AR, Brown G, Tait DM, Eccles SA, Swift RI (2001) Non-invasive methods of assessing angiogenesis and their value in predicting response to treatment in colorectal cancer. $\mathrm{Br} \mathrm{J}$ Surg 88: $1628-1636$

Goh V, Sanghera B, Wellsted DM, Sundin J, Halligan S (2009) Assessment of the spatial pattern of colorectal tumour perfusion estimated at perfusion CT using two-dimensional fractal analysis. Eur Radiol 19: $1358-1365$

Gonzalez-Garcia I, Sole RV, Costa J (2002) Metapopulation dynamics and spatial heterogeneity in cancer. Proc Natl Acad Sci USA 99: 13085-13089

Grigsby PW, Perez CA, Chao KS, Elbendary A, Herzog TJ, Rader JS, Mutch DG (1999) Lack of effect of tumor size on the prognosis of carcinoma of the uterine cervix Stage IB and IIA treated with preoperative irradiation and surgery. Int J Radiat Oncol Biol Phys 45: 645-651

Jackson A, O'Connor JP, Parker GJ, Jayson GC (2007) Imaging tumor vascular heterogeneity and angiogenesis using dynamic contrastenhanced magnetic resonance imaging. Clin Cancer Res 13: 3449-3459

Kinahan PE, Doot RK, Wanner-Roybal M, Bidaut LM, Armato SG, Meyer CR, McLennan G (2009) PET/CT assessment of response to therapy: tumor change measurement, truth data, and error. Transl Oncol 2: $223-230$

Klatte T, Patard JJ, de Martino M, Bensalah K, Verhoest G, de la Taille A, Abbou CC, Allhoff EP, Carrieri G, Riggs SB, Kabbinavar FF, Belldegrun AS, Pantuck AJ (2008) Tumor size does not predict risk of metastatic disease or prognosis of small renal cell carcinomas. J Urol 179: 1719-1726

Kumar R, Kuniyasu H, Bucana CD, Wilson MR, Fidler IJ (1998) Spatial and temporal expression of angiogenic molecules during tumor growth and progression. Oncol Res 10: $301-311$

Meyer CR, Armato SG, Fenimore CP, McLennan G, Bidaut LM, Barboriak DP, Gavrielides MA, Jackson EF, McNitt-Gray MF, Kinahan PE, Petrick N, Zhao B (2009) Quantitative imaging to assess tumor 
response to therapy: common themes of measurement, truth data, and error sources. Transl Oncol 2: 198-210

O'Connor JP, Carano RA, Clamp AR, Ross J, Ho CC, Jackson A, Parker GJ, Rose CJ, Peale FV, Friesenhahn M, Mitchell CL, Watson Y, Roberts C, Hope L, Cheung S, Reslan HB, Go MA, Pacheco GJ, Wu X, Cao TC, Ross S, Buonaccorsi GA, Davies K, Hasan J, Thornton P, del Puerto O, Ferrara N, van Bruggen N, Jayson GC (2009) Quantifying antivascular effects of monoclonal antibodies to vascular endothelial growth factor: insights from imaging. Clin Cancer Res 15: 6674-6682

O'Connor JP, Jackson A, Asselin MC, Buckley DL, Parker GJ, Jayson GC (2008) Quantitative imaging biomarkers in the clinical development of targeted therapeutics: current and future perspectives. Lancet Oncol 9: $766-776$

O'Connor JP, Jackson A, Parker GJ, Jayson GC (2007a) DCE-MRI biomarkers in the clinical evaluation of antiangiogenic and vascular disrupting agents. Br J Cancer 96: 189-195

O'Connor JP, Jayson GC, Jackson A, Ghiorghiu D, Carrington BM, Rose CJ, Mills SJ, Swindell R, Roberts C, Mitchell CL, Parker GJ (2007b) Enhancing fraction predicts clinical outcome following first-line chemotherapy in patients with epithelial ovarian carcinoma. Clin Cancer Res 13: 6130-6135

Orton MR, Miyazaki K, Koh DM, Collins DJ, Hawkes DJ, Atkinson D, Leach MO (2009) Optimizing functional parameter accuracy for breath-hold DCE-MRI of liver tumours. Phys Med Biol 54: 2197-2215

Parker GJ, Roberts C, Macdonald A, Buonaccorsi GA, Cheung S, Buckley DL, Jackson A, Watson Y, Davies K, Jayson GC (2006) Experimentally- derived functional form for a population-averaged high-temporalresolution arterial input function for dynamic contrast-enhanced MRI. Magn Reson Med 56: $993-1000$

Picchio M, Beck R, Haubner R, Seidl S, Machulla HJ, Johnson TD, Wester HJ, Reischl G, Schwaiger M, Piert M (2008) Intratumoral spatial distribution of hypoxia and angiogenesis assessed by 18F-FAZA and 125I-Gluco-RGD autoradiography. J Nucl Med 49: $597-605$

Robinson SP, McIntyre DJ, Checkley D, Tessier JJ, Howe FA, Griffiths JR, Ashton SE, Ryan AJ, Blakey DC, Waterton JC (2003) Tumour dose response to the antivascular agent ZD6126 assessed by magnetic resonance imaging. Br J Cancer 88: $1592-1597$

Rose CJ, Mills S, O'Connor JP, Buonaccorsi GA, Roberts C, Watson Y, Whitcher B, Jayson G, Jackson A, Parker GJ (2007) Quantifying heterogeneity in dynamic contrast-enhanced MRI parameter maps. Med Image Comput Comput Assist Interv 10: 376-384

Rose CJ, Mills SJ, O'Connor JP, Buonaccorsi GA, Roberts C, Watson Y, Cheung S, Zhao S, Whitcher B, Jackson A, Parker GJ (2009) Quantifying spatial heterogeneity in dynamic contrast-enhanced MRI parameter maps. Magn Reson Med 62: 488-499

Schroeder T, Yuan H, Viglianti BL, Peltz C, Asopa S, Vujaskovic Z, Dewhirst MW (2005) Spatial heterogeneity and oxygen dependence of glucose consumption in R3230Ac and fibrosarcomas of the Fischer 344 rat. Cancer Res 65: 5163-5171

Tofts PS (1997) Modeling tracer kinetics in dynamic Gd-DTPA MR imaging. J Magn Reson Imaging 7: $91-101$ 